\title{
Health Care Resource Utilization and Costs Associated with Corticosteroid Use in Patients with Castration-Resistant Prostate Cancer: An Administrative Claims Analysis
}

\author{
Neil M. Schultz, PharmD, MS; David F. Penson, MD; Samuel D. Wilson, PhD; Yan Song, PhD; \\ Hongbo Yang, PhD; Krishnan Ramaswamy, PhD; and Benjamin Lowentritt, MD
}

\begin{abstract}
BACKGROUND: Corticosteroids are used in the management of castrationresistant prostate cancer (CRPC) to reduce tumor-related symptoms because of CRPC therapies. Since corticosteroids have been associated with a range of toxicities, their use may increase the economic burden sustained by patients with CRPC. However, the economic impact of using corticosteroids in patients with CRPC has not been well characterized.
\end{abstract}

OBJECTIVE: To assess the effect of previous corticosteroid use on health care resource utilization (HRU) and health care costs among men with CRPC.

METHODS: Using administrative claims data (2007-2016), adult chemotherapy-naive patients who initiated CRPC treatment following surgical or medical castration were identified. Based on the cumulative corticosteroid dose during the 12 months before CRPC treatment initiation, patients were grouped into 4 cohorts: no corticosteroid ( $0 \mathrm{gm})$, low corticosteroid (<0.5 gm), medium corticosteroid (0.5-2.0 gm), and high corticosteroid (>2.0 gm). All-cause HRU and costs (2017 U.S. dollars) were compared between cohorts during the 1-year study period following CRPC treatment initiation using the no corticosteroid cohort as reference. Multivariable regression models were used to adjust for baseline covariates, including age, region, index year, Charlson Comorbidity Index score, presence of bone metastases, baseline all-cause $\mathrm{HRU}$, and corticosteroid-related clinical events during baseline.

RESULTS: 9,425 patients were included (no corticosteroid $=6,765$, low corticosteroid $=1,660$, medium corticosteroid $=655$, and high corticosteroid $=345$ ). On average, patients in the no corticosteroid cohort were older and had a lower baseline HRU and comorbidity burden than patients in the other 3 cohorts. During the study period, patients with corticosteroid exposure (across all corticosteroid cohorts) had significantly more inpatient admissions (high corticosteroid vs. no corticosteroid adjusted incidence rate ratio [IRR] $=1.56 ; P<0.001$ ), emergency department visits (high corticosteroid vs. no corticosteroid adjusted IRR $=1.30 ; P=0.001$ ), and outpatient visits (high corticosteroid vs. no corticosteroid adjusted IRR $=1.11$; $P<0.001)$. In addition, compared with the no corticosteroid cohort, patients with corticosteroid exposure had significantly higher monthly total costs (high corticosteroid vs. no corticosteroid adjusted difference $=\$ 2,600$; $P<0.001$ ), including medical service costs (high corticosteroid vs. no corticosteroid adjusted difference $=\$ 1,564 ; P<0.001$ ) and pharmacy costs (high corticosteroid vs. no corticosteroid adjusted difference $=\$ 825 ; P<0.001$ ).

CONCLUSIONS: Cumulative corticosteroid exposure before CRPC treatment initiation was associated with significantly higher HRU and costs. This increase in economic burden was more prominent among patients with annual cumulative corticosteroid doses of more than $2.0 \mathrm{gm}$. These results suggest that previous corticosteroid use may result in a higher economic burden among patients with CRPC.

J Manag Care Spec Pharm. 2019;25(8):889-97

Copyright $\odot 2019$, Academy of Managed Care Pharmacy. All rights reserved.

\section{What is already known about this subject}

Corticosteroids have been used in the management of castrationresistant prostate cancer (CRPC) for decades.

Corticosteroids have been associated with a number of toxicities, some severe and even life threatening, particularly if used at high doses or for a prolonged period of time.

\section{What this study adds}

Health care resource utilization (HRU) and costs were significantly higher in patients with CRPC exposed to corticosteroids in the year preceding initiation of a CRPC treatment compared with those not exposed to corticosteroids.

The increase in HRU and costs was more prominent among patients with previous annual cumulative doses of corticosteroids totaling more than $2.0 \mathrm{gm}$.

Previous corticosteroid use may result in a higher economic burden for patients with CRPC.

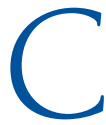

astration-resistant prostate cancer (CRPC) continues to grow and spread despite the use of surgical castration or androgen deprivation therapy to reduce serum testosterone to castrate levels. ${ }^{1}$ Approximately 10\%-20\% of patients develop CRPC within 5 years of being diagnosed with prostate cancer. ${ }^{2}$ At the time of CRPC diagnosis, about $84 \%$ of patients present with metastatic disease, while at least 5\% develop metastases within 2 years after diagnosis. ${ }^{2,3}$ The median overall survival of patients with CRPC has been estimated to be $<3$ years. ${ }^{3-5}$

For decades, the treatment of CRPC has involved chemotherapy and secondary hormonal therapy. ${ }^{1,6,7}$ However, the side effects of these therapies can be severe, including nausea, edema, and hypersensitivity reactions in the case of chemotherapy and acute adrenal crisis and apparent mineralocorticoid excess syndrome in the case of secondary hormonal therapy. ${ }^{4,8,9}$ To mitigate some of the side effects that accompany chemotherapy and secondary hormonal therapy, corticosteroids such as prednisone, hydrocortisone, and dexamethasone are typically used as concomitant treatment., ${ }^{4,9}$ Because of their anti-inflammatory properties, corticosteroids are also 


\section{Health Care Resource Utilization and Costs Associated with Corticosteroid Use in Patients with Castration-Resistant Prostate Cancer: An Administrative Claims Analysis}

used as a palliative treatment to relieve CRPC-related symptoms such as metastatic bone pain. . $^{4,9}$

Although corticosteroids are an integral part of the management of many diseases because of their beneficial clinical effects, they have also been associated with a number of toxicities, some severe and even life threatening, including adrenal suppression, diabetes, osteoporosis, infection, myopathy, hypertension, glaucoma, and cardiovascular disease.,10-12 In addition, prolonged exposure to corticosteroids has been linked to steroid dependency that, in the case of abrupt withdrawal, can result in severe side effects such as secondary adrenal insufficiency, myalgia, anorexia, nausea, fever, and asthenia., ${ }^{43,14}$ Because of the many clinical events that may develop following concomitant or palliative treatment with corticosteroids, sustained exposure to corticosteroids is likely to increase the health care resource utilization (HRU) and health care costs sustained by patients with CRPC. However, while some studies have found a positive association between the use of corticosteroids and increased HRU and costs in patients with several diseases, the economic impact of corticosteroid use on HRU and costs in patients with CRPC specifically remains unclear. ${ }^{11,15-17}$

Since most patients with CRPC are elderly, the effect of corticosteroids in this patient population may be particularly severe and, therefore, substantially contribute to increasing HRU and health care costs. ${ }^{18}$ One study reported more inpatient stays, higher monthly inpatient costs, and higher total health care costs in patients with CRPC with corticosteroid-sensitive comorbidities compared with those without corticosteroidsensitive comorbidities, but the data used were limited to 20072012, included only Veterans Health Administration claims, and did not consider different corticosteroid exposure levels. ${ }^{19}$

Given the evolving treatment landscape of CRPC, a more recent and comprehensive assessment of the economic implications of corticosteroid exposure in patients with CRPC is needed. This is particularly important as the use of corticosteroids remains common in clinical practice in the United States, despite the approval of several novel agents that do not require the use of corticosteroids for the treatment of metastatic CRPC (e.g., sipuleucel-T), nonmetastatic CRPC (e.g., apalutamide), or both (e.g., enzalutamide). ${ }^{20-22}$ Accordingly, using claims data, this study assessed the association between cumulative corticosteroid dose in the year before CRPC treatment initiation and HRU and health care costs among patients with CRPC in clinical practice in the United States.

\section{Methods}

\section{Data Source}

This study used data from the Truven Health Analytics MarketScan Commercial Claims and Encounters database, which covers employees and dependents aged 0-64 years, and the Medicare Supplemental database, which covers patients aged $\geq 65$ years with Medicare coverage, plus employer-paid commercial plans. Data were obtained from the period of January 1, 2007, to December 31, 2016. Data were fully deidentified and compliant with the Health Insurance Portability and Accountability Act (HIPAA). No institutional review board approval was required.

\section{Patient Selection and Study Design}

To be included in the analysis, male patients aged $\geq 18$ years were required to have $\geq 1$ medical claim with a diagnosis for prostate cancer based on the International Classification of Diseases, Ninth/Tenth Revision, Clinical Modification (ICD-9-CM code 185 or ICD-10-CM code C61) and a confirmed history of surgical or medical castration. Surgical castration was defined as unilateral or bilateral orchiectomy. Medical castration was defined as $\geq 6$ months of continuous androgen deprivation therapy with a luteinizing hormone-releasing hormone agonist or antagonist (leuprolide, goserelin, triptorelin, histrelin, or degarelix). To identify patients with CRPC, patients were further required to have initiated CRPC treatment after surgical or medical castration, including bicalutamide, flutamide, nilutamide, ketoconazole, enzalutamide, abiraterone acetate, cabazitaxel, docetaxel, radium-223, or sipuleucel-T.

Systemic use of the following corticosteroids was considered: betamethasone, budesonide, cortisone, dexamethasone, hydrocortisone, methylprednisolone, prednisolone, prednisone, and triamcinolone. Corticosteroid doses were standardized to prednisone-equivalent doses. Injectable corticosteroid treatments were not included in the analysis because they could not be converted to prednisone-equivalent doses and represented less than $1 \%$ of the corticosteroids in the current data. Patients were excluded if they had implausible corticosteroid doses corresponding to daily prednisone-equivalent doses of $>150 \mathrm{mg}$. Patients were also excluded if they had received any cytotoxic chemotherapy before the index date, defined as the initiation of CRPC treatment following surgical or medical castration. Continuous enrollment for $\geq 12$ months before and $\geq 3$ months after the index date was required. The study period was defined as the period from the index date to 1 year after the index date or the end of continuous enrollment or data availability, whichever occurred first. The use of corticosteroids was evaluated during the 12 months before the index date (the baseline period).

\section{Study Cohorts}

Patients were grouped into 4 cohorts based on the cumulative corticosteroid dose during the baseline period: the no corticosteroid cohort (patients who did not receive corticosteroids); the low corticosteroid cohort (patients who received a cumulative corticosteroid dose of $<0.5 \mathrm{gm}$, corresponding to $<1.4$ mg per day for 360 days); the medium corticosteroid cohort (patients who received a cumulative corticosteroid dose of 


\section{Health Care Resource Utilization and Costs Associated with Corticosteroid Use in Patients with Castration-Resistant Prostate Cancer: An Administrative Claims Analysis}

\section{FIGURE 1 Sample Selection Flowchart}

Male patients with $\geq 1$ medical claim in the Truven Health Analytics MarketScan database (2007-2016) with a diagnosis of prostate cancer $\mathrm{N}=929,144$

$\downarrow$

Patients with evidence of surgical or medical castration after the first prostate cancer diagnosis $\mathrm{n}=98,795$

Patients who initiated a new treatment for CRPC following surgical or medical castration.

The date of initiation or treatment (index treatment) is defined as the index date $n=15,223$

Patients with continuous enrollment for $\geq 12$ months before and $\geq 3$ months after the index date $n=10,564$

$\downarrow$

Patients aged $\geq 18$ years at the index date $n=10,564$

Patients who did not have claims for any chemotherapy before initiation of CRPC treatment $n=9,827$

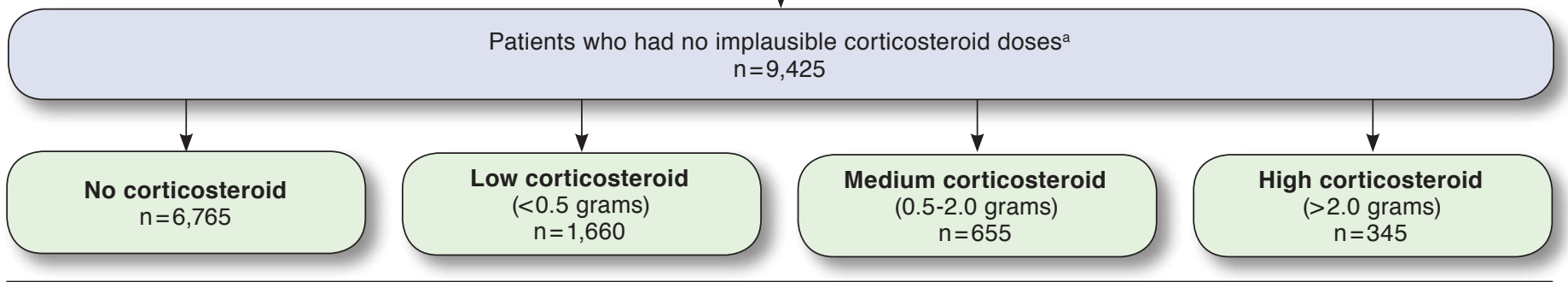

${ }^{a}$ Corticosteroids identified in the data included betamethasone, budesonide, cortisone, dexamethasone, hydrocortisone, methylprednisolone, prednisolone, prednisone, and triamcinolone.

$C R P C=$ castration-resistant prostate cancer.

0.5-2.0 gm, corresponding to 1.4-5.6 mg per day for 360 days); and the high corticosteroid cohort (patients who received a cumulative corticosteroid dose of $>2.0 \mathrm{gm}$, corresponding to $>5.6 \mathrm{mg}$ per day for 360 days). In the medical literature, there is no standardized definition of low, medium, and high corticosteroid usage. Therefore, in the current study, the cohorts were based on the cumulative corticosteroid dose distribution observed in the data (to ensure a sufficiently large sample size for each cohort), while making sure that the selected values of low, medium, and high cumulative corticosteroid doses were easily interpretable, as done in previous studies. ${ }^{11,23-25}$

\section{Study Outcomes}

All-cause HRU and direct health care costs were assessed during the study period. HRU included emergency department (ED) visits, outpatient visits, inpatient admissions, and days of hospitalization. Health care costs included medical service costs (inpatient, outpatient, and ED costs), pharmacy costs, and total costs. The costs were adjusted to 2017 U.S. dollars (USD) according to the medical care component of the Consumer Price Index.

\section{Statistical Analyses}

Baseline characteristics were summarized descriptively by cohort. Continuous variables were summarized using mean 


\section{Health Care Resource Utilization and Costs Associated with Corticosteroid Use in Patients with Castration-Resistant Prostate Cancer: An Administrative Claims Analysis}

\section{TABLE 1 Demographic and Clinical Baseline Characteristics by Cohort}

\begin{tabular}{|c|c|c|c|c|c|c|c|c|}
\hline \multirow{2}{*}{$\begin{array}{l}\text { Patient Characteristics } \\
\text { Age, years, mean (SD) }\end{array}$} & \multicolumn{2}{|c|}{$\begin{array}{c}\text { No } \\
\text { Corticosteroid } \\
\mathbf{n}=6,765\end{array}$} & \multicolumn{2}{|c|}{$\begin{array}{c}\text { Low } \\
\text { Corticosteroid } \\
(<0.5 \mathrm{gm}) \\
\mathrm{n}=1,660\end{array}$} & \multicolumn{2}{|c|}{$\begin{array}{c}\text { Medium } \\
\text { Corticosteroid } \\
(0.5-2.0 \mathrm{gm}) \\
\mathrm{n}=655\end{array}$} & \multicolumn{2}{|c|}{$\begin{array}{c}\text { High } \\
\text { Corticosteroid } \\
(>2.0 \mathrm{gm}) \\
\mathrm{n}=345\end{array}$} \\
\hline & 76.0 & $(10.5)$ & 73.7 & $(10.8)$ & 72.7 & $(10.6)$ & 71.0 & $(10.8)$ \\
\hline \multicolumn{9}{|l|}{ Insurance type, n (\%) } \\
\hline Comprehensive & 2,785 & $(41.2)$ & 579 & $(34.9)$ & 202 & $(30.8)$ & 96 & $(27.8)$ \\
\hline $\mathrm{PPO}$ & 2,785 & $(41.2)$ & 768 & $(46.3)$ & 322 & $(49.2)$ & 167 & $(48.4)$ \\
\hline HMO and other capitated plans & 695 & $(10.3)$ & 168 & $(10.1)$ & 68 & $(10.4)$ & 38 & $(11.0)$ \\
\hline Other & 500 & $(7.4)$ & 145 & $(8.7)$ & 63 & $(9.6)$ & 44 & $(12.8)$ \\
\hline Medicare supplemental coverage, n (\%) & 5,430 & $(80.3)$ & 1,214 & $(73.1)$ & 467 & $(71.3)$ & 219 & $(63.5)$ \\
\hline \multicolumn{9}{|l|}{ Year of index date, $\mathrm{n}(\%)$} \\
\hline $2007-2010$ & 2,208 & $(32.6)$ & 508 & $(30.6)$ & 207 & $(31.6)$ & 100 & $(29.0)$ \\
\hline $2011-2013$ & 2,809 & $(41.5)$ & 694 & $(41.8)$ & 283 & $(43.2)$ & 154 & $(44.6)$ \\
\hline $2014-2016$ & 1,748 & $(25.8)$ & 458 & $(27.6)$ & 165 & $(25.2)$ & 91 & $(26.4)$ \\
\hline \multicolumn{9}{|l|}{ CRPC treatments initiated on index date, ${ }^{a} \mathrm{n}(\%)$} \\
\hline Abiraterone & 614 & $(9.1)$ & 397 & $(23.9)$ & 118 & $(18.0)$ & 59 & $(17.1)$ \\
\hline Bicalutamide & 3,888 & $(57.5)$ & 408 & $(24.6)$ & 116 & $(17.7)$ & 34 & (9.9) \\
\hline Docetaxel & 473 & $(7.0)$ & 533 & $(32.1)$ & 268 & $(40.9)$ & 129 & $(37.4)$ \\
\hline Enzalutamide & 410 & (6.1) & 63 & (3.8) & 52 & $(7.9)$ & 59 & (17.1) \\
\hline Ketoconazole & 550 & (8.1) & 131 & $(7.9)$ & 48 & (7.3) & 29 & $(8.4)$ \\
\hline Otherb & 834 & $(12.3)$ & 130 & $(7.8)$ & 53 & $(8.1)$ & 35 & $(10.1)$ \\
\hline Cumulative corticosteroid dose during the baseline period, gm, mean (SD) & 0.0 & $(0.0)$ & 0.2 & $(0.1)$ & 1.1 & $(0.4)$ & 3.4 & $(2.6)$ \\
\hline \multicolumn{9}{|l|}{ Comorbidities during the baseline period } \\
\hline $\mathrm{CCI},{ }^{\mathrm{c}}$ mean $(\mathrm{SD})$ & 2.7 & $(1.2)$ & 2.9 & (1.3) & 3.0 & $(1.3)$ & 3.0 & $(1.4)$ \\
\hline Bone metastases corticosteroid-related conditions, n (\%) & 2,158 & $(31.9)$ & 863 & $(52.0)$ & 440 & $(67.2)$ & 262 & $(75.9)$ \\
\hline \multicolumn{9}{|l|}{ Cardiovascular disease } \\
\hline Hypertensive disease & 4,130 & $(61.0)$ & 1,019 & $(61.4)$ & 431 & $(65.8)$ & 200 & $(58.0)$ \\
\hline Ischemic heart disease & 1,930 & $(28.5)$ & 507 & $(30.5)$ & 188 & $(28.7)$ & 71 & $(20.6)$ \\
\hline Cerebrovascular disease & 859 & $(12.7)$ & 220 & $(13.3)$ & 82 & $(12.5)$ & 32 & $(9.3)$ \\
\hline Heart failure & 637 & $(9.4)$ & 170 & $(10.2)$ & 68 & $(10.4)$ & 41 & $(11.9)$ \\
\hline Diabetes & 1,922 & $(28.4)$ & 431 & $(26.0)$ & 168 & $(25.6)$ & 90 & $(26.1)$ \\
\hline Peripheral vascular disease & 960 & $(14.2)$ & 242 & $(14.6)$ & 89 & (13.6) & 44 & $(12.8)$ \\
\hline Fractures & 480 & $(7.1)$ & 163 & $(9.8)$ & 78 & $(11.9)$ & 42 & $(12.2)$ \\
\hline Osteoporosis & 423 & $(6.3)$ & 106 & $(6.4)$ & 47 & $(7.2)$ & 28 & $(8.1)$ \\
\hline Peptic ulcers and related complications & 60 & $(0.9)$ & 12 & $(0.7)$ & 7 & $(1.1)$ & 10 & $(2.9)$ \\
\hline \multicolumn{9}{|l|}{ Mental health conditions } \\
\hline Depression & 324 & $(4.8)$ & 108 & $(6.5)$ & 52 & $(7.9)$ & 26 & $(7.5)$ \\
\hline Bipolar disorder & 21 & $(0.3)$ & 4 & $(0.2)$ & 5 & $(0.8)$ & 1 & $(0.3)$ \\
\hline \multicolumn{9}{|l|}{ Infections } \\
\hline Pneumonia & 264 & (3.9) & 117 & $(7.0)$ & 63 & $(9.6)$ & 43 & $(12.5)$ \\
\hline Septicemia/bacteremia & 127 & (1.9) & 52 & $(3.1)$ & 23 & $(3.5)$ & 23 & $(6.7)$ \\
\hline \multicolumn{9}{|l|}{ Endocrine disorders } \\
\hline Abnormal glucose level & 334 & $(4.9)$ & 90 & $(5.4)$ & 33 & $(5.0)$ & 22 & (6.4) \\
\hline Adrenal disorder & 51 & $(0.8)$ & 19 & $(1.1)$ & 13 & $(2.0)$ & 8 & $(2.3)$ \\
\hline Abnormal weight gain & 26 & $(0.4)$ & 8 & $(0.5)$ & 3 & $(0.5)$ & 1 & $(0.3)$ \\
\hline
\end{tabular}

and standard deviation (SD); categorical variables were summarized using counts and proportions.

The HRU and cost outcomes of the patients in the low, medium, and high corticosteroid cohorts were compared with those of the patients in the no corticosteroid cohort. Unadjusted and adjusted comparisons were conducted. The HRU incidence rate was calculated per month per patient; monthly rates allowed for comparisons between patients with different follow-up times using all available data. Incidence rate ratios (IRRs) and 95\% confidence intervals (CIs) were estimated using generalized linear models with a Poisson distribution.

Health care costs were calculated per month per patient by dividing the total costs accrued during the study period by the duration of follow-up from the index date. Costs were 


\section{Health Care Resource Utilization and Costs Associated with Corticosteroid Use in Patients with Castration-Resistant Prostate Cancer: An Administrative Claims Analysis}

\section{TABLE 1 Demographic and Clinical Baseline Characteristics by Cohort (continued)}

\begin{tabular}{|c|c|c|c|c|}
\hline Patient Characteristics & $\begin{array}{c}\text { No } \\
\text { Corticosteroid } \\
\mathrm{n}=6,765\end{array}$ & $\begin{array}{c}\text { Low } \\
\text { Corticosteroid } \\
(<0.5 \mathrm{gm}) \\
\mathrm{n}=1,660 \\
\end{array}$ & $\begin{array}{c}\text { Medium } \\
\text { Corticosteroid } \\
(0.5-2.0 \mathrm{gm}) \\
\mathrm{n}=655\end{array}$ & $\begin{array}{c}\text { High } \\
\text { Corticosteroid } \\
(>2.0 \mathrm{gm}) \\
\mathbf{n}=345\end{array}$ \\
\hline \multicolumn{5}{|l|}{ Health care resource utilization, mean (SD) } \\
\hline Inpatient admissions & $(0.57)$ & $(0.72)$ & $(0.84)$ & $(0.86)$ \\
\hline Days of hospitalization (among all patients) & $(4.38)$ & $(5.87)$ & $(6.84)$ & $(8.71)$ \\
\hline Emergency department visits & $0.70 \quad(1.48)$ & $(1.85)$ & $(2.34)$ & $1.28 \quad(2.55)$ \\
\hline Outpatient visits & $26.10 \quad(19.28)$ & $33.74 \quad(19.00)$ & $39.76 \quad(21.34)$ & $42.48 \quad(22.01)$ \\
\hline Health care costs (2017 USD), ${ }^{\mathrm{d}}$ mean (SD) & $\mathrm{n}=6,070$ & $\mathrm{n}=1,492$ & $\mathrm{n}=587$ & $\mathrm{n}=307$ \\
\hline Total health care costs & $31,558(52,534)$ & $45,170(57,449)$ & $64,839(76,539)$ & $93,346(87,667)$ \\
\hline Medical service cost & $27,479(51,251)$ & $39,830(55,795)$ & $54,489(72,386)$ & $69,203(81,099)$ \\
\hline Inpatient admissions & $6,210(25,894)$ & $8,987(33,509)$ & $14,143 \quad(47,098)$ & $14,498(33,130)$ \\
\hline Emergency department visits & $770 \quad(3,122)$ & $1,256 \quad(3,594)$ & $1,840 \quad(8,529)$ & $1,839 \quad(4,501)$ \\
\hline Outpatient visits & $20,499 \quad(39,713)$ & $29,587(38,850)$ & $38,507 \quad(46,702)$ & $52,866(64,951)$ \\
\hline Pharmacy cost & $4,079 \quad(9,718)$ & $5,340 \quad(8,879)$ & $10,350 \quad(22,432)$ & $24,144(34,725)$ \\
\hline \multicolumn{5}{|c|}{$\begin{array}{l}\text { aCombination therapies were included in CRPC treatments initiated at index date and included bicalutamide and ketoconazole, radium-223 and enzalutamide, } \\
\text { abiraterone and bicalutamide, and docetaxel and abiraterone. } \\
\text { bOther CRPC treatments included cabazitaxel, flutamide, nilutamide, radium-223, and sipuleucel-T. } \\
\text { 'The CCI score has been modified to exclude prostate cancer and metastatic disease. } \\
\text { dThe cost analyses excluded } 969 \text { patients who were on capitated plans; these patients constituted about } 10 \% \text { of the total sample. } \\
C C I=\text { Charlson Comorbidity Index; CRPC= castration-resistant prostate cancer; HMO=health maintenance organization; PPO=preferred provider organization; } \\
\text { SD=standard deviation; USD =U.S. dollars. }\end{array}$} \\
\hline
\end{tabular}

calculated per month to account for varying follow-up times between patients. Generalized linear models with a Tweedie distribution (a compound Poisson-gamma distribution) were used to compare the cost outcomes between patients. ${ }^{26-28}$ To ensure that the service costs were accurately represented, the cost analyses only included patients who were not on capitated insurance plans.

Adjusted HRU and cost analyses controlled for the following: patient age at the index date, geographic region, baseline Charlson Comorbidity Index score, year of index date, baseline all-cause and prostate cancer-related inpatient admissions and outpatient visits, and presence of bone metastasis, fractures, mental health conditions, infections, endocrine disorders, cardiovascular disease, and peptic ulcers and related complications during the baseline period.

\section{Results}

$\overline{\text { A total of 9,425 patients were included in the analysis (Figure 1). }}$ Of these, 6,765 were in the no corticosteroid cohort, 1,660 in the low corticosteroid cohort, 655 in the medium corticosteroid cohort, and 345 in the high corticosteroid cohort. For each index year, the relative distribution of patients across cohorts was comparable.

\section{Baseline Characteristics}

On average at baseline, patients in the no corticosteroid cohort were older than patients in the low, medium, and high corticosteroid cohorts (mean 76.0 vs. 71.0-73.7 years; Table 1).
The majority of patients in all cohorts (98\%) received medical castration treatment; only $2 \%$ received surgical castration. More than half of the patients in the no corticosteroid cohort initiated bicalutamide as a new CRPC treatment following castration, while about one third of patients in the low, medium, and high corticosteroid cohorts initiated docetaxel following castration. In addition, a higher proportion of corticosteroid nonusers initiated antiandrogen therapies (including bicalutamide, enzalutamide, flutamide, and nilutamide) than those who used corticosteroids (70.0\% vs. 29.0\%-32.7\%; Table 1).

When considering comorbidities at baseline, patients in the no corticosteroid cohort had a lower mean Charlson Comorbidity Index score than patients in the other 3 cohorts (means 2.7 vs. 2.9-3.0; Table 1). Over 70\% of patients in each cohort had cardiovascular disease, including hypertensive disease, ischemic heart disease, cerebrovascular disease, and heart failure. A larger proportion of patients in the low, medium, and high corticosteroid cohorts had bone metastases (52\%-76\%) compared with patients in the no corticosteroid cohort (32\%). This trend was also observed for other comorbidities, including fractures, infections, depression, and adrenal disorder (Table 1).

At baseline, HRU and costs presented a clear increasing trend with higher cumulative corticosteroid dose. Specifically, the average number of inpatient admissions and ED visits during baseline was twice as high in the high corticosteroid cohort as in the no corticosteroid cohort ( 0.57 vs. 0.26 and 1.28 vs. 0.70 , respectively), while average annual health care costs among patients in the high corticosteroid cohort were almost 


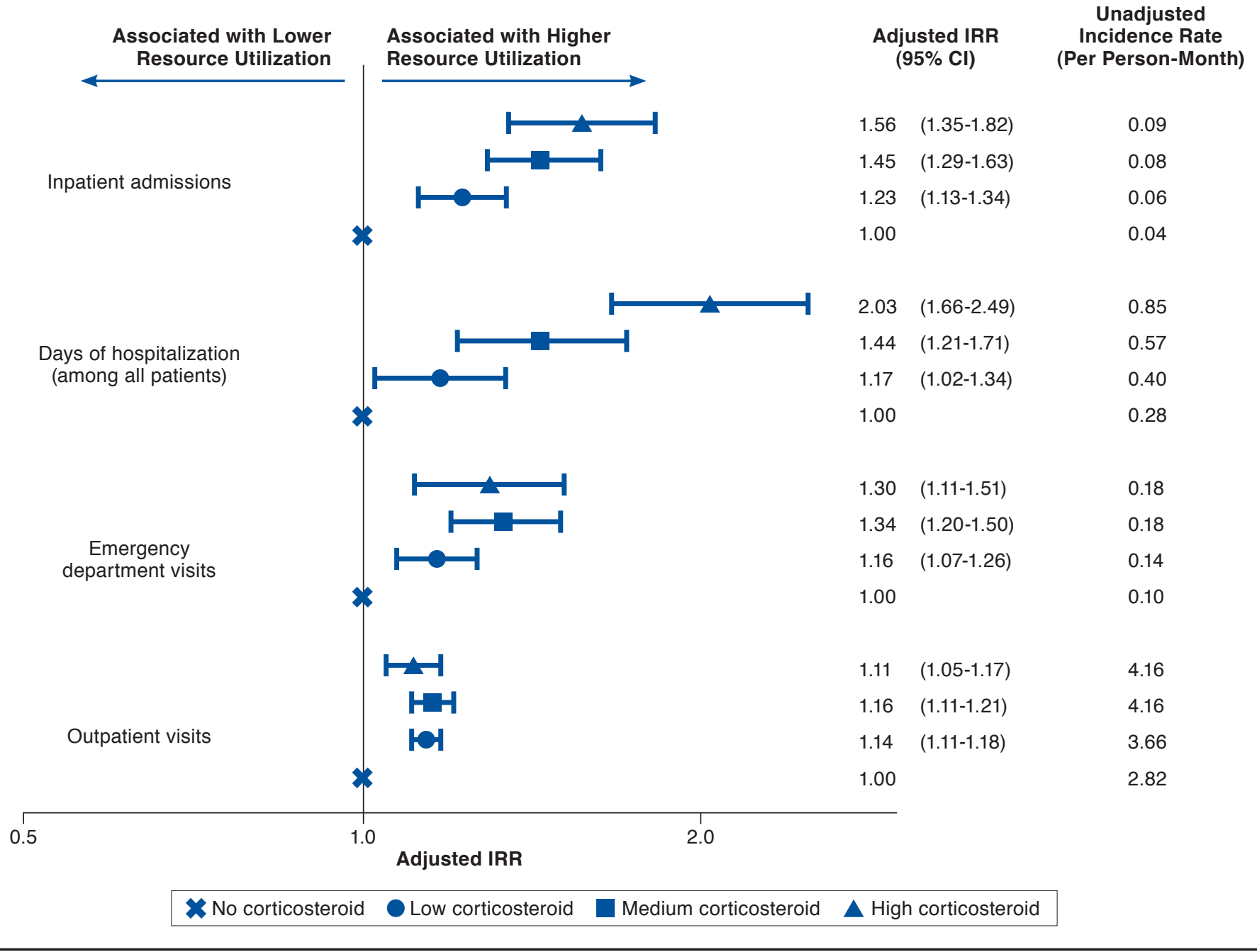

aAn estimate is statistically significant whenever the confidence interval does not include 1.0 (does not cross the vertical axis). Adjustments were made for the following baseline covariates: age, index year, geographic region, baseline all-cause and prostate cancer-related inpatient admission and outpatient visits, Charlson Comorbidity Index score, and presence of bone metastasis, fractures, mental health conditions, infections, endocrine disorders, cardiovascular disease, and peptic ulcers and related complications.

$C I=$ confidence interval; $I R R=$ incidence rate ratio.

3 times as high as the costs among patients in the no corticosteroid cohort ( $\$ 93,346$ vs. $\$ 31,558$; Table 1$)$.

\section{HRU and Costs}

Compared with the no corticosteroid cohort, patients in the low, medium, and high corticosteroid cohorts had significantly more inpatient admissions, ED visits, and outpatient visits (Figure 2). The adjusted IRRs (95\% CIs) for the high corticosteroid cohort versus the no corticosteroid cohort were 1.56 (1.35-1.82) for inpatient admissions, 1.30 (1.11-1.51) for ED visits, and 1.11 (1.05-1.17) for outpatient visits.
The mean monthly health care costs (standard deviation [SD]) among patients in the high corticosteroid cohort were about twice as high as those among patients in the no corticosteroid cohort $(\$ 14,836[\$ 11,824]$ vs. $\$ 7,417[\$ 10,266]$; Table 2$)$. Patients in the low, medium, and high corticosteroid cohorts had significantly higher medical service and pharmacy costs than patients in the no corticosteroid cohort. In particular, patients in the high corticosteroid cohort had the highest incremental monthly pharmacy cost (adjusted difference compared with the no corticosteroid cohort $=\$ 825 ; P<0.001)$ and monthly inpatient and outpatient costs (adjusted differences 


\section{Health Care Resource Utilization and Costs Associated with Corticosteroid Use in Patients with Castration-Resistant Prostate Cancer: An Administrative Claims Analysis}

\section{TABLE 2 Health Care Costs (2017 USD) Following the Index Date}

\begin{tabular}{|c|c|c|c|c|c|c|c|c|c|c|c|c|}
\hline \multicolumn{13}{|c|}{ Difference in Monthly Cost ${ }^{a, b}$} \\
\hline & \multicolumn{6}{|c|}{ Unadjusted } & \multicolumn{6}{|c|}{ Adjusted $^{c}$} \\
\hline & \multicolumn{2}{|c|}{$\begin{array}{c}\text { Low } \\
\text { Corticosteroid- } \\
\text { No Corticosteroid }\end{array}$} & \multicolumn{2}{|c|}{$\begin{array}{c}\text { Medium } \\
\text { Corticosteroid- } \\
\text { No Corticosteroid } \\
\end{array}$} & \multicolumn{2}{|c|}{$\begin{array}{c}\text { High } \\
\text { Corticosteroid- } \\
\text { No Corticosteroid } \\
\end{array}$} & \multicolumn{2}{|c|}{\begin{tabular}{|c|} 
Low \\
Corticosteroid- \\
No Corticosteroid \\
\end{tabular}} & \multicolumn{2}{|c|}{\begin{tabular}{|c|} 
Medium \\
Corticosteroid- \\
No Corticosteroid \\
\end{tabular}} & \multicolumn{2}{|c|}{$\begin{array}{c}\text { High } \\
\text { Corticosteroid- } \\
\text { No Corticosteroid } \\
\end{array}$} \\
\hline & Diff & $P$ Valued & Diff & $P$ Value ${ }^{d}$ & Diff & $P$ Valued & Diff & $P$ Valued & Diff & $P$ Valued & Diff & $P$ Value $^{\mathrm{d}}$ \\
\hline Total health care cost & 2,959 & $<0.001$ & 4,385 & $<0.001$ & 7,418 & $<0.001$ & 1,591 & $<0.001$ & 1,435 & $<0.001$ & 2,600 & $<0.001$ \\
\hline Medical service cost & 2,039 & $<0.001$ & 3,420 & $<0.001$ & 5,896 & $<0.001$ & 723 & $<0.001$ & 706 & 0.020 & 1,564 & $<0.001$ \\
\hline Inpatient admissions & 561 & $<0.001$ & 1,006 & $<0.001$ & 2,455 & $<0.001$ & 324 & $<0.001$ & 403 & 0.003 & 1,249 & $<0.001$ \\
\hline Emergency department visits & 65 & $<0.001$ & 197 & $<0.001$ & 165 & $<0.001$ & 32 & 0.003 & 115 & $<0.001$ & 56 & 0.009 \\
\hline Outpatient visits & 1,412 & $<0.001$ & 2,217 & $<0.001$ & 3,277 & $<0.001$ & 442 & 0.003 & 374 & $0.092^{\mathrm{e}}$ & 669 & 0.030 \\
\hline Pharmacy cost & 920 & $<0.001$ & 965 & $<0.001$ & 1,522 & $<0.001$ & 620 & $<0.001$ & 427 & $<0.001$ & 825 & $<0.001$ \\
\hline \multicolumn{13}{|c|}{$\begin{array}{l}\text { aThe cost analyses excluded } 969 \text { patients who were on } c \\
b \text { Cost differences and } P \text { values were estimated using gen } \\
\text { varying follow-up times between patients. } \\
\text { cAdjusted models controlled for patient age, index year, } \\
\text { Charlson Comorbidity Index score, and presence of bon } \\
\text { ulcers and related complications. } \\
{ }^{d} \text { These } P \text { values are statistically significanct }(P<0.05) \text {. } \\
\text { eThis value is not statistically significant }(P \geq 0.05) \text {. } \\
\text { Diff = difference; USD =U.S. dollars. }\end{array}$} \\
\hline
\end{tabular}

compared with the no corticosteroid cohort $=\$ 1,249$ and $\$ 669$; $P<0.001$ and $P=0.030$, respectively; Table 2 ).

\section{Discussion}

Although corticosteroids are often used as a concomitant or palliative treatment in patients with CRPC, $, 8,9$ their economic impact on the CRPC patient population has not been well characterized in the literature. To better understand the economic burden of using corticosteroids in patients with CRPC, this study used claims data to assess the association between cumulative corticosteroid dose in the year before initiating CRPC treatment and HRU and health care costs among patients with CRPC in clinical practice in the United States.

The results of this study showed that patients with CRPC treated with corticosteroids before initiation of CRPC treatment had higher HRU and increased medical and pharmacy costs than patients with CRPC not previously treated with corticosteroids. Notably, the HRU and cost burden increased with cumulative corticosteroid dose. In particular, patients in the low, medium, and high corticosteroid cohorts had significantly more inpatient admissions, ED visits, and outpatient visits compared with the no corticosteroid cohort. In addition, patients with baseline corticosteroid exposure had significantly higher medical service and pharmacy costs compared with patients without baseline corticosteroid exposure.

Very few studies have evaluated the economic implications of systemic corticosteroid exposure. Nonetheless, those few studies have reported a positive correlation between corticosteroid exposure and increased HRU and costs in patients with several diseases, including systemic lupus erythematosus, ${ }^{10,29}$ sarcoidosis,$^{30}$ and asthma. ${ }^{15,31}$ In patients with systemic lupus erythematosus, for instance, yearly incremental costs relative to the no corticosteroid cohort have been found to range from $\$ 5,700$ for patients receiving $<7.5 \mathrm{mg}$ per day prednisone equivalent to $\$ 29,000$ (2012 USD) for patients receiving > $15 \mathrm{mg}$ per day prednisone equivalent. ${ }^{29}$

In the case of CRPC, only 1 study assessing the economic burden associated with corticosteroids was identified in the literature. In that study, which considered patients with CRPC using corticosteroids concomitantly with cabazitaxel, docetaxel, or abiraterone acetate, patients with corticosteroid-sensitive comorbidities were found to have more inpatient stays (20.5\% vs. $12.9 \% ; P=0.033)$, higher monthly inpatient costs $(\$ 1,157$ vs. $\$ 342 ; P<0.0001)$, and higher total costs $(\$ 5,725$ vs. $\$ 4,772 ; P=0.036)$ than those without corticosteroid-sensitive comorbidities. ${ }^{19}$ However, the study was limited to claims data from the Veterans Health Administration database spanning only 2007-2012 and did not consider different cumulative corticosteroid doses. The present study represents a more comprehensive and current assessment of the HRU and health care costs associated with varying cumulative corticosteroid exposure in the year before initiating CRPC treatment.

The increase in HRU and health care costs in patients with CRPC previously treated with corticosteroids observed in this study may be attributable to the management of the clinical complications associated with exposure to corticosteroids. In some studies, reducing the dose of corticosteroids was linked to a reduction in the number of corticosteroid-related 


\section{Health Care Resource Utilization and Costs Associated with Corticosteroid Use in Patients with Castration-Resistant Prostate Cancer: An Administrative Claims Analysis}

complications and costs. ${ }^{11,15}$ For instance, in a 2011 study assessing the economic burden of corticosteroid-related complications in patients with different diseases (including rheumatoid arthritis, acute lymphoblastic leukemia, and asthma, but not prostate cancer), Sarnes et al. ${ }^{11}$ estimated that reducing the daily corticosteroid dose from high (defined as $>30 \mathrm{mg}$ per day prednisone equivalent) to low (defined as $<7.5 \mathrm{mg}$ per day prednisone equivalent) would avoid 96 fractures per 10,000 people, resulting in a cost reduction of $\$ 1.76$ million per 10,000 people, and 19.4 myocardial infarctions per 10,000, resulting in a cost reduction of over $\$ 500,000$ per 10,000 people (2009 USD). Similarly, a 2016 study assessing the cost of corticosteroid exposure in patients with severe asthma found that reducing systemic corticosteroid exposure from a dose of $>5-10 \mathrm{mg}$ per day to $<5 \mathrm{mg}$ per day prednisone equivalent would result in a cost reduction of nearly $\$ 4$ million per 10,000 people (2014 USD) by avoiding bone- and muscle-related complications such as fractures..$^{15}$ Studies estimating the cost of corticosteroid-related clinical events in the CRPC patient population is needed to better understand the reasons for the increase in HRU and costs observed in this study.

The current study fills an important gap in the estimation of the economic burden of previous corticosteroid exposure among patients with CRPC, highlighting the significant increase in HRU and health care costs that patients sustain when they receive corticosteroids before CRPC treatment initiation. This suggests that CRPC treatments that do not require the use of corticosteroids may help reduce the HRU and cost burden for patients by limiting the overall exposure to corticosteroids and, consequently, reducing the incidence of corticosteroid-related clinical events. This is particularly important given that the treatment landscape for CRPC continues to evolve and now includes regimens that do not require corticosteroids. As more evidence pertaining to the adverse effects and costs associated with corticosteroids in patients with CRPC is presented in future studies, prescribing habits may change, and over time, exposure to corticosteroids may be reduced or even avoided, whenever possible. Such changes, and any effect they may have on HRU and costs, will need to be monitored in future real-world studies.

It is worth noting that patients who used corticosteroids before initiating CRPC treatment may continue to use corticosteroids after treatment initiation, as $82 \%$ of the patient sample in this study did. Continuing corticosteroid use during CRPC treatment may worsen the economic burden sustained by patients as they are likely to be exposed to increasingly higher cumulative doses of corticosteroid and suffer from corticosteroid-related side effects. CRPC treatments that do not require corticosteroid use may help reduce the overall exposure to corticosteroids. Additional studies are needed to evaluate whether CRPC treatments that do not require corticosteroid use would be associated with a lower HRU and cost burden.

\section{Limitations}

The results of this study should be considered in light of some limitations. First, in the claims data, patients with CRPC were identified using proxy measures (required medical or surgical castration and initiation of a CRPC treatment), given that there is no specific ICD code for a CRPC diagnosis.

Second, injectable corticosteroids were not included because of the impossibility of converting them into a standardized prednisone-equivalent dose. Thus, the calculated cumulative corticosteroid dose may not capture all corticosteroid use among patients. However, injectable corticosteroids made up $<1 \%$ of pharmacy claims in the current data, and their use is not expected to systematically differ across cohorts.

Third, claims data may not reflect the actual drug use of a patient, since they do not capture medical services obtained outside of a patient's plan, do not include over-the-counter drugs (e.g., nonsteroidal anti-inflammatory drugs), and may not include all prescribed drugs (e.g., drug samples); therefore, pharmacy costs may have been underestimated.

Fourth, since the analysis was conducted among commercially insured patients, the results may not be generalizable to patients covered by public insurance plans. Further, although commercial and Medicare supplemental claims data were used to include patients of all ages, the claims did not include medical services completely covered by Medicare (e.g., skilled nursing facility services after discharge).

Finally, because of the observational nature of this study, the possibility of bias (e.g., confounding or selection bias) cannot be excluded. Specifically, residual confounding from the characteristics that were not documented in the claims data (e.g., baseline disease severity) may exist. To the extent possible, we controlled for the observed baseline imbalance among cohorts by considering proxies of disease severity (e.g., baseline prostate cancer-related HRU) and using multivariable regression modeling.

\section{Conclusions}

The current study found that exposure to corticosteroids in patients with CRPC before initiating CRPC treatment was associated with significantly increased HRU and costs. This increase in economic burden was more prominent among patients with annual cumulative corticosteroid doses of $>2.0 \mathrm{gm}$. Previous corticosteroid use may result in a higher economic burden for patients with CRPC. 


\section{Health Care Resource Utilization and Costs Associated with Corticosteroid Use in Patients with Castration-Resistant Prostate Cancer: An Administrative Claims Analysis}

\section{Authors}

NEIL M. SCHULTZ, PharmD, MS, and SAMUEL D. WILSON, PhD, Astellas Pharma, Northbrook, Illinois. YAN SONG, PhD, and HONGBO YANG, PhD, Analysis Group, Boston, Massachusetts. DAVID F. PENSON, MD, Vanderbilt University Medical Center, Nashville, Tennessee; KRISHNAN RAMASWAMY, PhD, Pfizer, New York, New York; and BENJAMIN LOWENTRITT, MD, Chesapeake Urology, Towson, Maryland.

AUTHOR CORRESPONDENCE: Neil M. Schultz, PharmD, Astellas Pharma, 1 Astellas Way, Northbrook, IL 60062. Tel.: 224.205.5415; E-mail: neil.schultz@astellas.com.

\section{DISCLOSURES}

This study was funded by Astellas Pharma (Northbrook, IL) and Medivation, a Pfizer Company (San Francisco, CA), the codevelopers of enzalutamide. The study sponsor was involved in the study design, data interpretation, and review. All authors contributed to the development of the manuscript and maintained control over the final content. Schultz and Wilson are employed by Astellas Pharma. Schultz owns stock in Gilead Sciences and Shire. Song and Yang are employed by Analysis Group, which received consultancy fees from Astellas Pharma. Ramaswamy is employed by Pfizer, and Lowentritt is employed by Chesapeake Urology and has served as a speaker and consultant for Astellas Pharma, Pfizer, Bayer, Dendreon, and Janssen.

A synopsis of the current research was presented in poster format at the AMCP Managed Care \& Specialty Pharmacy Annual Meeting 2019, which took place in San Diego, CA, on March 25-28, 2019.

\section{ACKNOWLEDGMENTS}

Analytical support was provided by Iryna Bocharova, Emily Gao, and Ellie Fuqua, employees of Analysis Group; medical writing assistance was provided by Cinzia Metallo, PhD, an employee of Analysis Group; and editorial assistance was provided by Caitlin Watson, $\mathrm{PhD}$, and Jane Beck from Complete HealthVizion, all funded by the study sponsors.

\section{REFERENCES}

1. Hotte SJ, Saad F. Current management of castrate-resistant prostate cancer. Curr Oncol. 2010;17(Suppl 2):S72-79.

2. Heidenreich A, Pfister D, Merseburger A, Bartsch G. Modern management of castration-resistant prostate cancer. Eur Oncol Haematol. 2013;9(1):34-41.

3. Kirby M, Hirst C, Crawford ED. Characterising the castration-resistant prostate cancer population: a systematic review. Int J Clin Pract. 2011;65(11):1180-92

4. De Santis M, Saad F. Practical guidance on the role of corticosteroids in the treatment of metastatic castration-resistant prostate cancer. Urology. 2016;96:156-64.

5. Huang X, Chau CH, Figg WD. Challenges to improved therapeutics for metastatic castrate resistant prostate cancer: from recent successes and failures. J Hematol Oncol. 2012;5:35.

6. Teply BA, Hauke RJ. Chemotherapy options in castration-resistant prostate cancer. Indian J Urol. 2016;32(4):262-70.

7. Saad F, Fizazi K. Androgen deprivation therapy and secondary hormone therapy in the management of hormone-sensitive and castration-resistant prostate cancer. Urology. 2015;86(5):852-61.

8. Dorff TB, Crawford ED. Management and challenges of corticosteroid therapy in men with metastatic castrate-resistant prostate cancer. Ann Oncol. 2013;24(1):31-38

9. Ndibe C, Wang CG, Sonpavde G. Corticosteroids in the management of prostate cancer: a critical review. Curr Treat Options Oncol. 2015;16(2):6.
10. Shah M, Chaudhari S, McLaughlin TP, et al. Cumulative burden of oral corticosteroid adverse effects and the economic implications of corticosteroid use in patients with systemic lupus erythematosus. Clin Ther. 2013;35(4):486-97.

11. Sarnes E, Crofford L, Watson M, Dennis G, Kan H, Bass D. Incidence and U.S. costs of corticosteroid-associated adverse events: a systematic literature review. Clin Ther. 2011;33(10):1413-32.

12. Liu D, Ahmet A, Ward L, et al. A practical guide to the monitoring and management of the complications of systemic corticosteroid therapy. Allergy Asthma Clin Immunol. 2013;9(1):30.

13. Margolin L, Cope DK, Bakst-Sisser R, Greenspan J. The steroid withdrawal syndrome: a review of the implications, etiology, and treatments. J Pain Symptom Manage. 2007;33(2):224-28.

14. Alves C, Robazzi TC, Mendonça M. Withdrawal from glucocorticosteroid therapy: clinical practice recommendations. J Pediatr (Rio J). 2008;84(3):192-202. 15. Dalal AA, Duh MS, Gozalo L, et al. Dose-response relationship between long-term systemic corticosteroid use and related complications in patients with severe asthma. J Manag Care Spec Pharm. 2016;22(7):833-47. Available at: https://www.jmcp.org/doi/10.18553/jmcp.2016.22.7.833.

16. Kabadi S, Yeaw J, Bacani AK, et al. Healthcare resource utilization and costs associated with long-term corticosteroid exposure in patients with systemic lupus erythematosus. Lupus. 2018;27(11):1799-809.

17. Rice JB, White AG, Johnson M, et al. Healthcare resource use and cost associated with varying dosages of extended corticosteroid exposure in a U.S. population. J Med Econ. 2018;21(9):846-52.

18. American Cancer Society. Key statistics for prostate cancer. Revised January 8, 2019. Available at: https://www.cancer.org/cancer/prostate-cancer/about/key-statistics.html. Accessed May 17, 2019.

19. Bui CN, Wang L, Baser O. Resource utilization and use of life-extending therapies and corticosteroids in prostate cancer patients with corticosteroidsensitive comorbidities. Curr Med Res Opin. 2014;30(11):2355-64.

20. Chong JT, Oh WK, Liaw BC. Profile of apalutamide in the treatment of metastatic castration-resistant prostate cancer: evidence to date. Onco Targets Ther. 2018;11:2141-47.

21. Gupta S, Carballido E, Fishman M. Sipuleucel-T for therapy of asymptomatic or minimally symptomatic, castrate-refractory prostate cancer: an update and perspective among other treatments. Onco Targets Ther. 2011;4:79-96.

22. Hussain M, Fizazi K, Saad F, et al. Enzalutamide in men with nonmetastatic, castration-resistant prostate cancer. N Engl J Med. 2018;378(26):2465-74. 23. Curtis JR, Westfall AO, Allison J, et al. Population-based assessment of adverse events associated with long-term glucocorticoid use. Arthritis Rheum. 2006;55(3):420-26.

24. Varas-Lorenzo C, Rodriguez LA, Maguire A, Castellsague J, PerezGutthann S. Use of oral corticosteroids and the risk of acute myocardial infarction. Atherosclerosis. 2007;192(2):376-83.

25. van Staa TP, Leufkens HG, Abenhaim L, Zhang B, Cooper C. Oral corticosteroids and fracture risk: relationship to daily and cumulative doses. Rheumatology (Oxford). 2000;39(12):1383-89.

26. Jørgensen B, Paes De Souza MC. Fitting Tweedie's compound Poisson model to insurance claims data. Scand Actuar J. 1994;(1):69-93.

27. Kaas R. Compound Poisson distribution and GLM's-Tweedie's distribution. In: De Schepper A, Dhaene J, Reynaerts H, Schoutens W, Van Goethem P, Vanmaele M, eds. 3rd Actuarial and Financial Mathematics Day. Wetteren, Belgium: Universa Press; 2005:3-12.

28. Klinker F. Generalized linear mixed models for ratemaking: a means of introducing credibility into a generalized linear model setting. Casualty Actuarial Society E-Forum. 2011;2(Winter):1-25. Available at: https://www. casact.org/pubs/forum/llwforumpt2/Klinker.pdf. Accessed May 25, 2019. 29. Chen SY, Choi CB, Li Q, et al. Glucocorticoid use in patients with systemic lupus erythematosus: association between dose and health care utilization and costs. Arthritis Care Res (Hoboken). 2015;67(8):1086-94.

30. Ligon CB, Judson MA. Impact of systemic corticosteroids on healthcare utilization in patients with sarcoidosis. Am J Med Sci. 2011;341(3):196-201. 31. Manson SC, Brown RE, Cerulli A, Vidaurre CF. The cumulative burden of oral corticosteroid side effects and the economic implications of steroid use. Respir Med. 2009;103(7):975-94. 